\title{
Steroid-induced psychosis as a manifestation of ectopic ACTH secretion from metastatic poorly differentiated neuroendocrine tumour
}

Sviatlana Zhyzhneuskaya', Rasha Mukhtar', Simon Ashwell', Sath Nag'

1 Endocrinology Department, The James Cook University Hospital, Middlesbrough

\author{
South Tees Hospitals $\mathbf{N H}$ \\ NHS Foundation Trust
}

Case Report

- 65 year old fisherman presented with altered mental status, agitation, generalised oedema, dyspnoea, liver impairment and severe persistent hypokalaemia $(2.2$ $\mathrm{mmol} / \mathrm{L})$.

- Past history: COPD, IPF (on steroids since May 2012), ex-smoker.

- Physical exam: deeply tanned, Cushingoid facies, severe proximal myopathy

- Medications prior to admission: Prednisolone 40mg OD, Furosemide-40mg OD, Inhalers, Lanzoprasole.

- In a view of clinical and biochemical features of steroid access the Endocrine referral was made.

Investigations

- Patient had following abnormal Initial Investigations endocrine tests: cortisol$3118 \mathrm{nmol} / \mathrm{l}, \mathrm{ACTH}-632 \mathrm{ng} / \mathrm{l}$, total chromogranin A-480u/l, 5-HIAA-551umol/24h.

- CT Thorax/Abdomen showed multiple liver metastasis, pulmonary fibrosis, RUL nodule, enlarged parathracheal, subcarinal lymph nodes, bilateral adrenal hypertrophy.

- CT head/MRI of pituitary were normal.

- Liver biopsy confirmed the presence of poorly differentiated (very high Ki67 index-90\%) small cell neuroendocrine tumour which positively stained for TTF1, CK7, CD56, AE1/3, chromogranin, synaptophysin.

- Positive staining for TTF1 and CK7 are sought to be in favour of primary lung lesion.

- The diagnosis of poorly differentiated metastatic small cell neuroendocrine tumour with ectopic ACTH secretion was made.

\section{Case Resolution}

- Patient was started on Metyrapone 1g 4 hourly. Ketoconazole 200mg OD was added later.

- His cortisol and liver function had initially improved and potassium normalised.

- Oncology team put him on three months course of carboplatin with etoposide. Further investigations suggested for ?Carcinoid in a form of Octreotide scan were not recommended by Oncology Department due to its relative insensitivity in the settings of poorly differentiated neuroendocrine tumour.

- Patient passed away just in four months since presentation despite intensive chemotherapy.
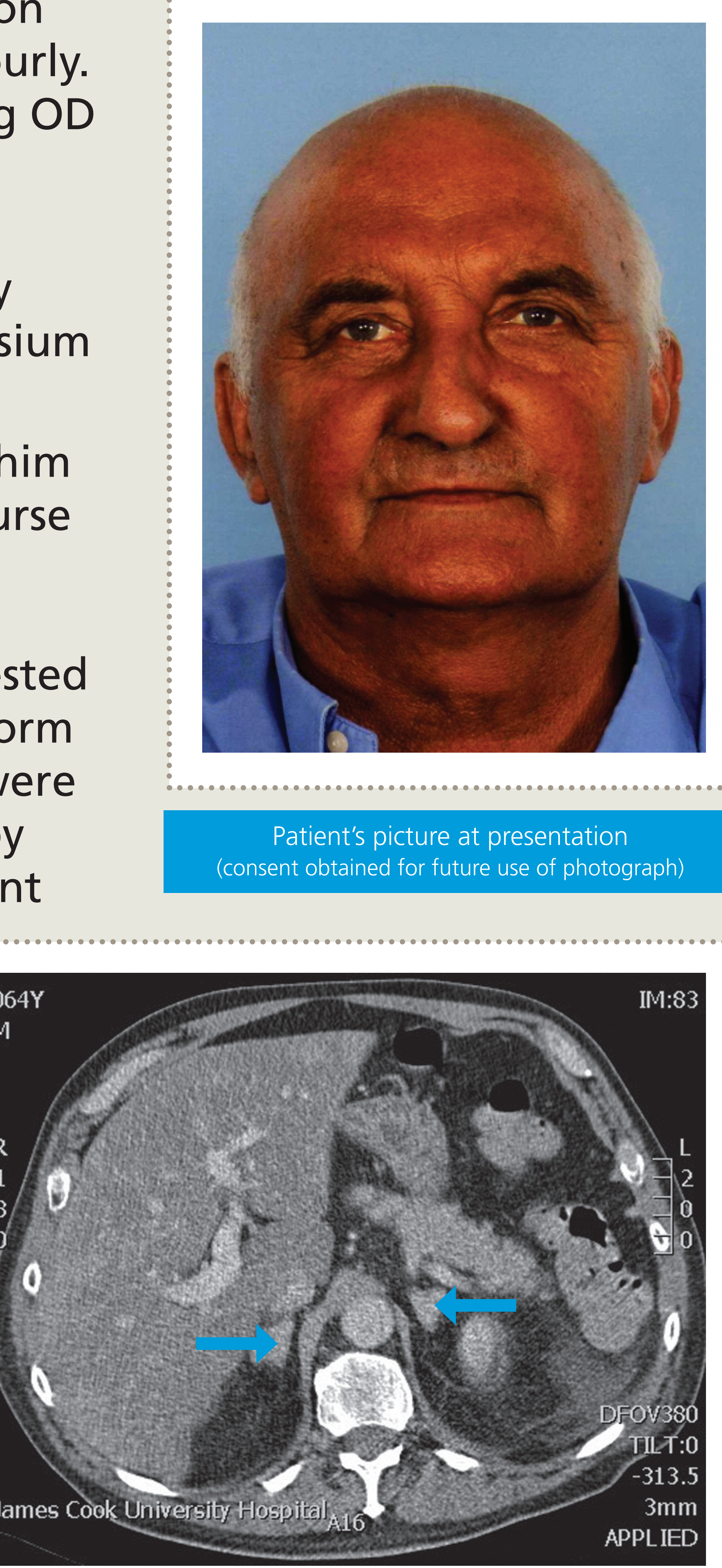

CT abdomen (arrows point towards bilateral bulky adrenal glands)

\section{Conclusion}

We reported this case because of the interesting clinical presentation of an ectopic ACTH secretion exacerbated by exogenous steroid intake. This case also shows the importance of good differential diagnosis of hypokalaemia. Treatment options were limited due to the aggressive nature of the tumour. 